\title{
Arbeit als Kinderarzt im ländlichen Ostafrika
}

Reto Villiger

\section{Erfahrungen aus einem Programm}

\section{für HIV-positive Kinder und Mütter in Tansania}

Es bestehen keine Interessensverbindungen.

Korrespondenz:

Dr. med. Reto Villiger Facharzt für Kinderund Jugendmedizin FMH Kinderarztpraxis im Institut für Arbeitsmedizin

Kreuzweg 3

CH-5400 Baden

Tel. 0562054464

Fax 0562057616

reto.villiger@arbeitsmedizin.ch
Malerisch am Fusse eines Hügelzuges gelegen, umgeben von Jacarandabäumen und Bananenstauden, liegt das Dareda Hospital $200 \mathrm{~km}$ südwestlich von Arusha im Hochland Nordtansanias. Eine rotbraune Schotterpiste zieht dem Hügelzug entlang und verbindet die weitläufige Gemeinde Daredas mit den Nachbardörfern, bis sie schliesslich in den Distrikthauptort Babati mündet. Die Mehrzahl der Einheimischen sind

einfache Bauern. Sie wohnen in traditionellen, rechteckigen, aus der rotbraunen Erde gefertigten und mit Stroh oder Bananenblättern gedeckten Häusern. Einige können sich auch Wellblechdächer leisten. Wasser wird aus den nahegelegenen Wasserstellen geholt und jeden Morgen in grossen Plastikkübeln, die die Mädchen geschickt auf ihren Köpfen balancierend tragen, ins eigene Heim gebracht. Am Strassenrand entdeckt man kleine Buben, deren Kleider die Farbe der staubigen Erde angenommen haben und die mit einem Holzstock die Tierherden vorantreiben. Die Menschen hier auf dem Lande leben einfach.

In Dareda arbeitete ich die vergangenen zwei Jahre für die Organisation SolidarMed als Leiter der Kinderstation und als technischer Berater des HIV-Programms. Meine Arbeit umfasste primär das gesamte Tätigkeitsfeld der Pädiatrie. Neben dieser allgemeinpädiatrischen Tätigkeit kam ich aber mit dem speziellen Auftrag nach Dareda, für den Aufbau eines pädiatrischen HIV-Programms zu sorgen. Eine Sprechstunde für HIV-Positive existierte bereits, jedoch wurden bisher wie in vielen anderen Spitälern Tansanias keine Kinder betreut. Zwar existierten HIV-positive Kinder, doch starben sie unbemerkt, bevor man die Diagnose überhaupt stellen konnte, an einer vordergründig anderen Krankheit.

Die Organisation eines HIV-Programms aus kinderärztlicher Sicht macht aus mehreren Gründen Sinn: Kinder sind das schwächste Glied in der afrikanischen Gesellschaft, sie sind gesundheitlich wie auch sozial vulnerabel und kaum jemand setzt sich politisch für sie ein. Dieser Umstand wurde schon vor Jahren von internationalen Organisationen erkannt. Ihre Gelder erreichen aber kaum je die eigentliche Zielpopulation, die Kinder. Auf die HIV-positiven Kinder übertragen heisst dies, dass auch jetzt noch die Mehrheit der süd- und ostafrikanischen Spitäler kaum Kinder mit HIV-Medikamenten behandeln.

Aus kinderärztlicher Sicht liegt der Hauptfokus eines HIV-Programms in der Vorbeugung der Mutter-Kind-Übertragung (prevention of mother to child transmission, PMTCT), da damit die Neuansteckung eines Kindes mit all ihren gesundheitlichen, sozialen und ökonomischen Folgen verhindert werden kann. Die Fokussierung auf die (werdenden) Mütter ist auch deshalb ein sehr wirksames Instrument, weil sowohl Mütter wie auch Kinder im Gesundheitswesen Tansanias relativ einfach zugänglich sind: Die Mehrheit von ihnen erscheint nämlich sogar in den ruralen Gebieten in den Vorsorgekliniken für Schwangere und Kleinkinder, wo sie gratis untersucht und geimpft werden. Somit ist eine gesundheitliche «Erschliessung» der ganzen Familie über die (werdenden) Mütter und Kleinkinder möglich. Eine gezielte Schulung und Stär-

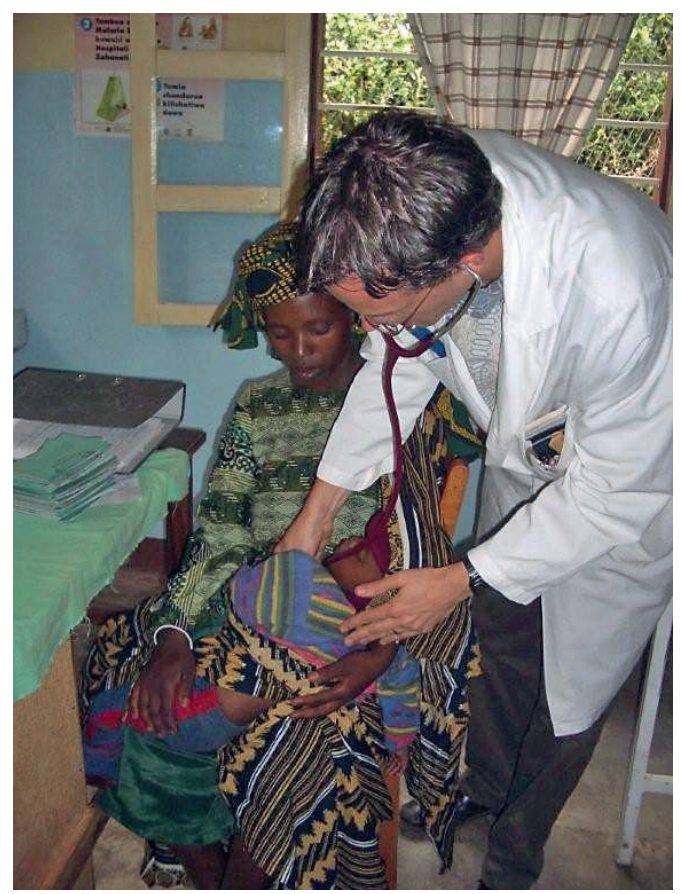

Untersuchung eines Kindes. 
kung der jungen Frauen soll einhergehen mit dem Erkennen vulnerabler Individuen, wie es HIV-positive Frauen sind. Denn die Frauen bilden innerhalb der tansanischen Gesellschaft eine Schlüsselrolle im Streben nach einer nachhaltigen Entwicklung.

Sodann war der wichtigste Ansatz eine starke Gewichtung des PMTCT innerhalb des HIV-Programms von Dareda. Als eine der ersten Massnahmen im Aufbau des Programms wurde eine routinemässige HIV-Testung der schwangeren Frauen in der vorgeburtlichen Vorsorgeklinik (Antenatal Clinic, ANC) eingeführt. Das Ziel war klar: möglichst viele der schwangeren Frauen einem HIV-Test unterziehen. Innert Kürze konnte erreicht werden, dass fast $100 \%$ der in der Vorsorgeklinik erschienenen Frauen geschult

\section{Abbildung 1}

Anzahl Geburten im Dareda Hospital vor und nach Beginn des PMTCT-Programms und Anzahl Frauen in der Maternité mit bekanntem HIV-Status (inkl. Prozentsatz der Frauen mit bekanntem HIV-Status am Entlassungstag)x.

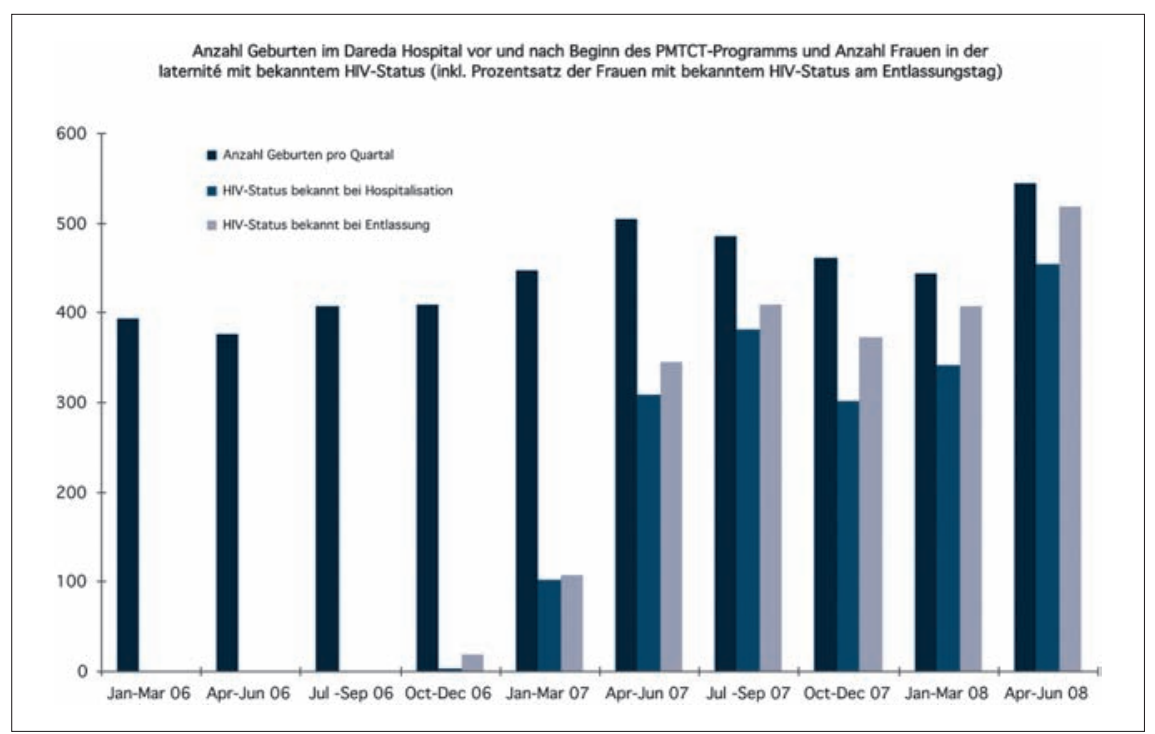

Abbildung 2

Standortbestimmung für alle ins PMTCT-Programm involvierten Kinder per Juni 2008.

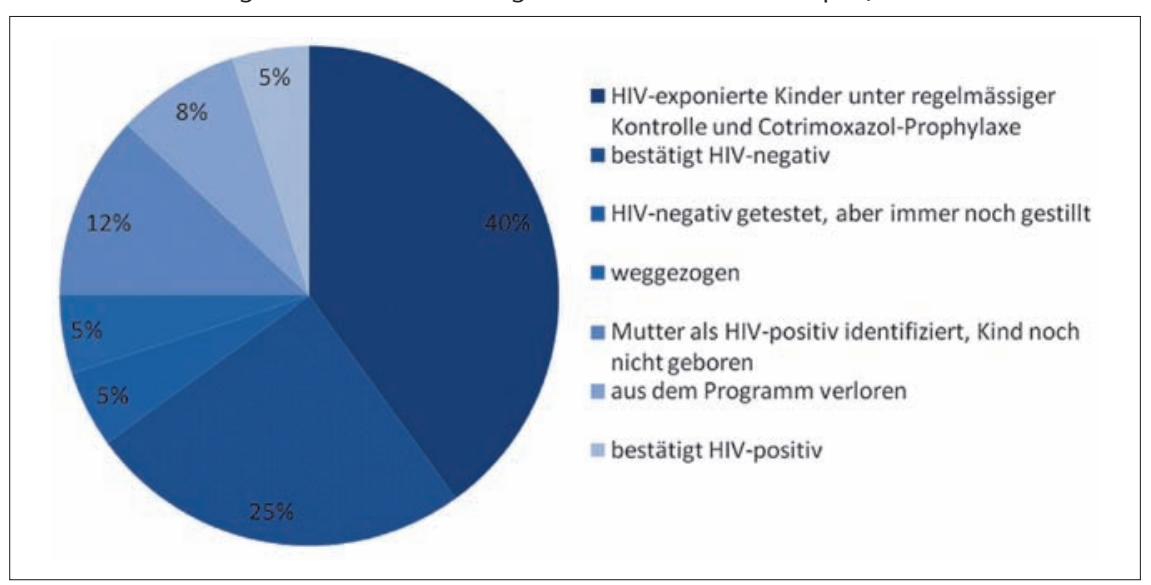

und getestet wurden. Somit war die Akzeptanz des Tests bei den Schwangeren unerwartet hoch. Wir konnten feststellen, dass die Schwierigkeiten anderer Spitäler im Lande, die mit deutlich sinkenden Zahlen von ANC-Patienten nach Einführung eines HIV-Routinetests konfrontiert waren, bei uns nicht eintrafen. Tatsächlich war die Anzahl der ANC-Besuche vor und nach Implementierung des Tests in etwa gleich und ist sie auch jetzt noch, während wir fortfahren, fast jede Schwangere zu testen.

Als zweite Massnahme wurde in der Entbindungs- und Wöchnerinnenstation des DaredaSpitals eine routinemässige Überprüfung des HIVStatus aller gebärenden Frauen eingeführt. Dies konnte anhand der Vorsorge- und Geburtskarte jeder Frau erfolgen. Bei einem unklaren Status wurde die Frau dazu motiviert, einen HIV-Test (möglichst bald) während des Spitalaufenthalts durchzuführen. Dadurch konnte im Verlauf der Projektperiode erreicht werden, dass fast alle Frauen, die zur Geburt im Dareda-Spital erschienen waren, ihren HIV-Status kannten.

Die Anzahl Entbindungen im Dareda-Spital wurde durch die Einführung des HIV-Routinetests in der Vorgeburtsklinik bzw. in der Gebärabteilung nicht tangiert, wie dies Abbildung 1 illustriert. Überdies zeigt Abbildung 1, dass nach Einführung des HIV-Routinetests in der ANC die Zahl der gebärenden Frauen mit bekanntem HIVStatus in der Gebärabteilung stetig zunahm. Auch dies bestätigt, dass die schwangeren Frauen keineswegs wegen der Einführung des HIV-Routinetests das Dareda-Spital mieden. Trotz der weitverbreiteten Stigmatisierung von HIV-Positiven in der Region ist also die Akzeptanz der Schwangeren gegenüber dem Test gross.

Natürlich ist die Kenntnis des HIV-Status einer schwangeren bzw. gebärenden Frau nur der erste Schritt in der Prävention. Es mussten also klare Strukturen aufgebaut werden, aus denen ersichtlich ist, welche präventiven Massnahmen im Falle eines positiven Testresultats eingeleitet werden müssen. Dazu wurden einfache Flussdiagramme errichtet, die für die einzelnen Abteilungen adaptiert wurden. So wurde denn z. B. eine frisch entdeckte HIV-positive Schwangere nach Besprechung des Resultats in der Antenatal Clinic (Screeningort) von einer der Pflegefachfrauen am selben Tag ins Gebäude der HIV-Klinik eskortiert. Dort wurde gemeinsam mit Fachleuten des HIVTeams eine Krankengeschichte erhoben und eine klinische Untersuchung durchgeführt. So konnte eine bessere Einbindung in die HIV-Klinik erreicht werden. Gleichzeitig konnte ein intensives gemeinsames Beratungsgespräch stattfinden. Anhand der Resultate der Erstkonsultation konnte 
jeweils in einer Zweitkonsultation der Behandlungs- bzw. Präventionsplan besprochen werden.

Auch wenn es anfangs schwierig war, die HIVpositiven Schwangeren zur Teilnahme am Programm zu motivieren, konnte nach einigen Monaten die Rückkehrrate deutlich gesteigert werden. Es wurden spezielle Anreize für die Zielgruppe geschaffen: Rückerstattung von Transportkosten zur Teilnahme an den monatlichen Konsultationen im Spital und Direktabgabe von Nahrungsmitteln.

Nicht alle HIV-positiven Schwangeren sind zur Geburt im Dareda-Spital erschienen. Einige haben zu Hause oder in einem Gesundheitszentrum geboren und waren somit nur beschränkt für eine Prophylaxe zugänglich. Trotzdem zeigt die Auswertung der Krankengeschichten der bei uns registrierten PMTCT-Patientinnen, dass die meisten Kinder von HIV-positiven Müttern per Juni 2008 unter regelmässiger Kontrolle in unserem Spital standen (Abb. 2). Zudem bekamen 92\% der registrierten Mütter eine medikamentöse Pro- phylaxe (i.d.R. Nevirapin-Einzeldosis). Davon hatten nur $8 \%$ zu Hause geboren, nahmen aber die Medikamente selbständig ein, so dass mindestens ein Teil der vorbeugenden Massnahmen erfüllt werden konnte. Weitere $8 \%$ hatten zu Hause geboren und die Medikamente nicht eingenommen. Bei ihnen konnte also überhaupt keine Prophylaxe durchgeführt werden. Sie konnten aber im Verlauf der Zeit über das System der Home-based-Care-Freiwilligenhelfer in den Dörfern gefunden und wieder in die Klinik zurückgebracht werden. So wurde zumindest eine enge Begleitung von Mutter und Kind nach der Geburt möglich.

Das PMTCT-Programm von Dareda ist nun in eine zweite Phase getreten, die wiederum von einem Schweizer Kinderarzt geleitet wird: die Dezentralisierung. Und Dezentralisierung ist hier in zweifachem Sinne zu verstehen: geographisch vom Grossen zum Kleinen und innerfamiliär vom Kleinen zum Grossen.

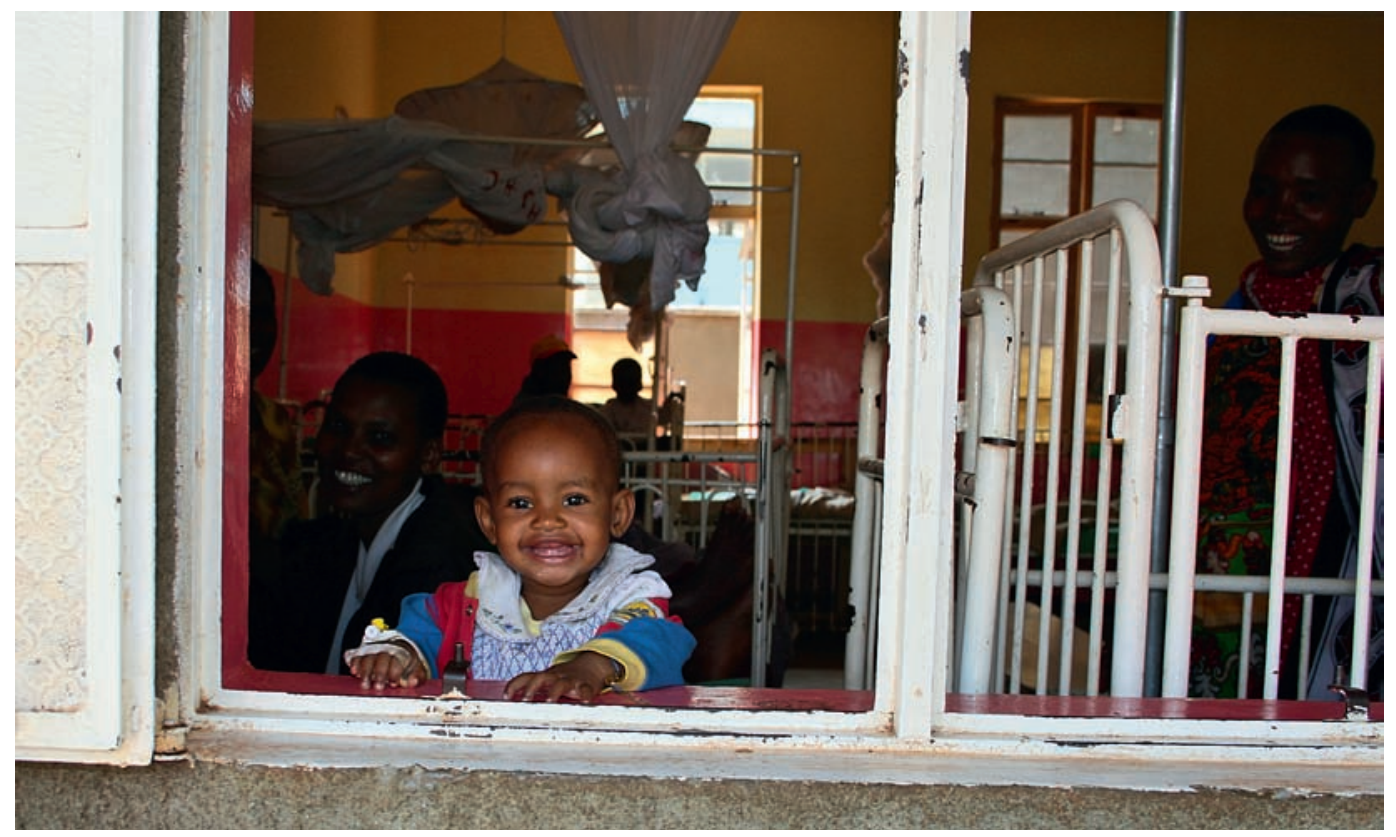

Genesenes Kind in der Kinderabteilung. 